\title{
Electrocardiographic and electrophysiological characteristics of idiopathic ventricular arrhythmias with acute successful ablation at the left ventricle basal inferoseptum near the mitral annulus
}

\author{
Chengye $\mathrm{Di}^{1}$, Peng $\mathrm{Gao}^{1}$, qun wang ${ }^{1}$, yanxi wu ${ }^{1}$, and Wenhua $\mathrm{Lin}^{1}$ \\ ${ }^{1}$ TEDA International Cardiovascular Hospital
}

November 19, 2020

\begin{abstract}
Introduction: We sought to clarify the electrocardiographic and electrophysiological characteristics of ventricular arrhythmias (VAs), including idiopathic ventricular tachycardia (VT) and premature ventricular contractions (PVCs), with acute successful radiofrequency catheter ablation (RFCA) at the left ventricle basal inferoseptum near the mitral annulus (LV-BIS-MA). Methods and Results: Twenty-five patients with acute successful RFCA at the LV-BIS-MA were included in this study. The S wave amplitudes on lead III during VAs were $1.54 \pm 0.38 \mathrm{mV}$, significantly larger than that on lead II $(0.55 \pm 0.19 \mathrm{mV})$ and aVF $(1.04 \pm 0.31 \mathrm{mV})(\mathrm{P}<0.01)$. The RFCA start-to-effect time was $10.2 \pm 5.8$ seconds $(\mathrm{s})$ in 21 patients $(84.0 \%)$. In the remaining 4 patients $(16.0 \%)$, the mean duration of successful RFCA was not well determined due to infrequent nature of clinical VAs during ablation. Trans-septal approach were utilized in all the 25 cases. Intra-cardiac echocardiography (ICE) showed that the ablation catheter tip was underneath the anterior leaflet of the mitral valve via the reversed C-curve technique. Early (within 3 days) and late (one-year) recurrence rates were $4.0 \%$ (one patient) and $12.0 \%$ (three patients), respectively. No complications occurred during RFCA or the one-year follow up. CONCLUSION: LV-BIS-MA VAs are a subgroup of idiopathic VAs with distinctive ECG and EP features. RFCA via a transseptal approach using a reversed $\mathrm{C}$ curve technique is effective for better identification and targeting the areas of VAs origin and ICE showed that the ablation catheter tip was underneath the anterior leaflet of the mitral valve.
\end{abstract}

\section{Hosted file}

manuscript.2020.11.17.pdf available at https://authorea.com/users/376998/articles/493839$\%$ CE\%Blectrocardiographic-and-electrophysiological-characteristics-of-idiopathicventricular-arrhythmias-with-acute-successful-ablation-at-the-left-ventricle-basalinferoseptum-near-the-mitral-annulus 

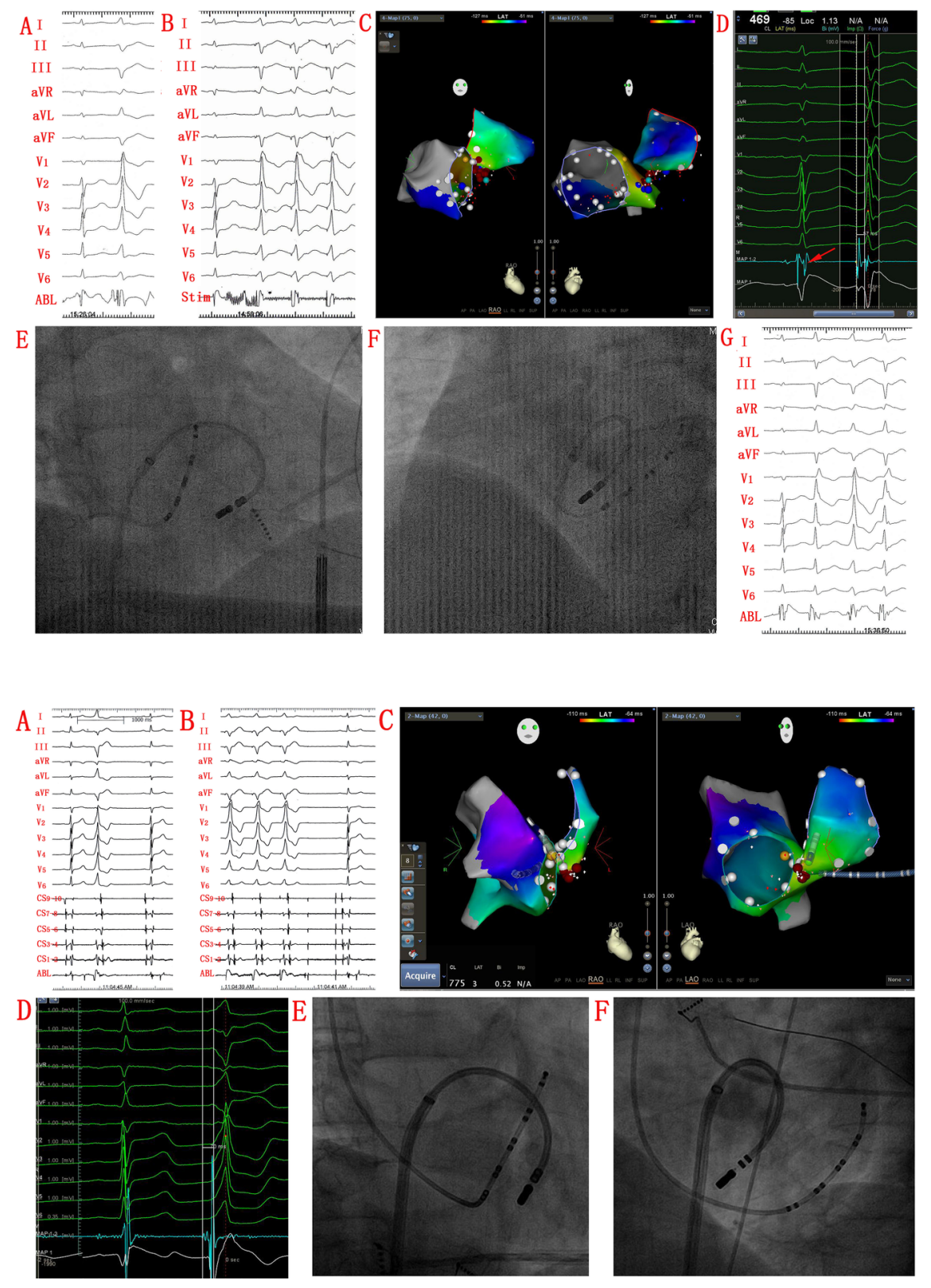

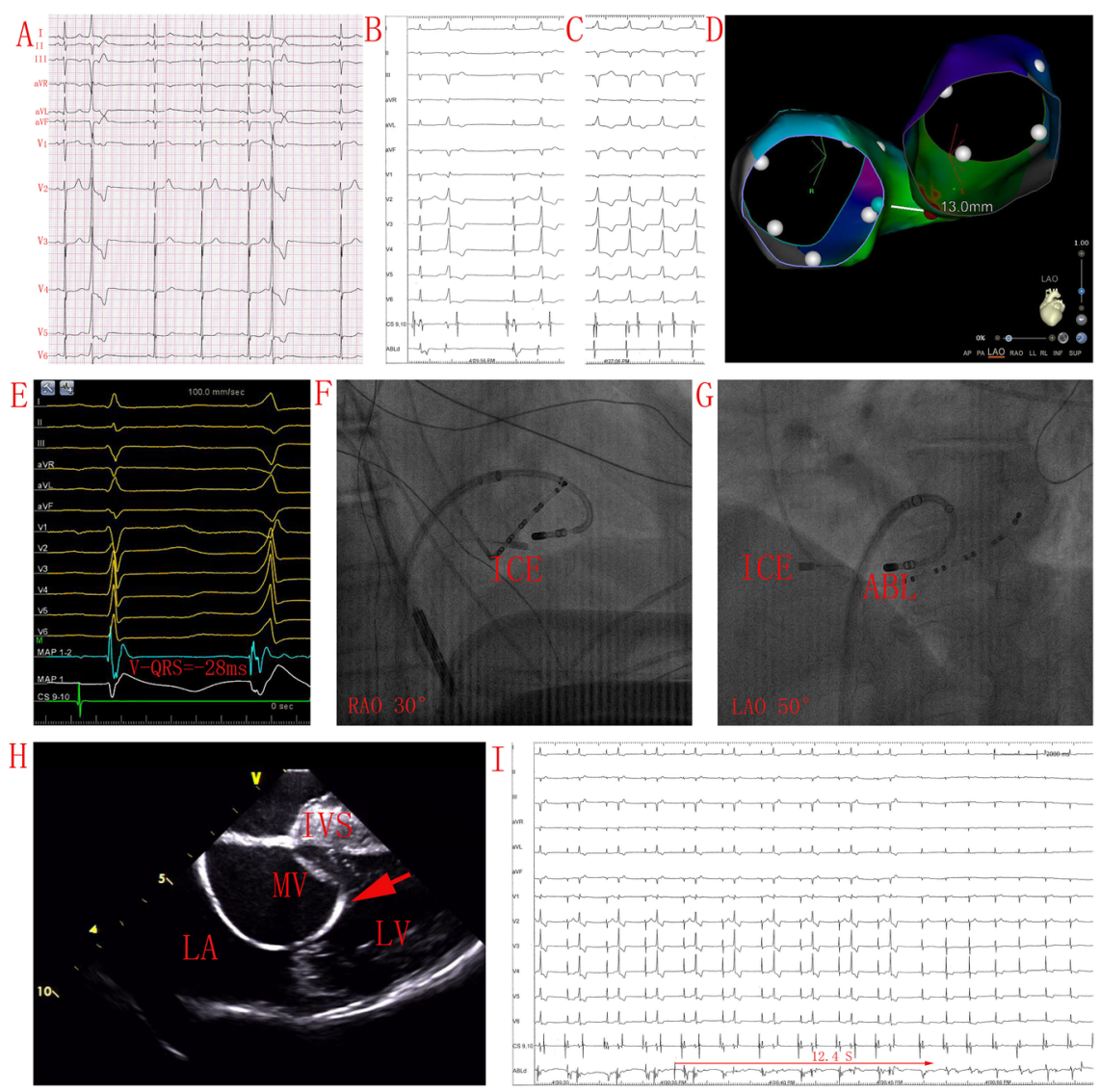


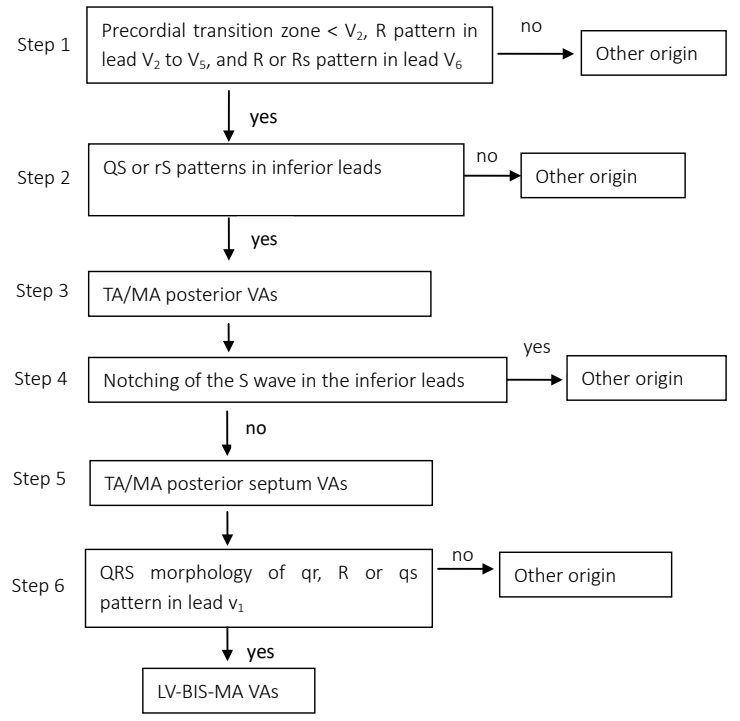

\section{Hosted file}

Table 1.2020.11.1.pdf available at https://authorea.com/users/376998/articles/493839$\% \mathrm{CE} \% \mathrm{~B} 51$ ectrocardiographic-and-electrophysiological-characteristics-of-idiopathicventricular-arrhythmias-with-acute-successful-ablation-at-the-left-ventricle-basalinferoseptum-near-the-mitral-annulus

\section{Hosted file}

Table 2.2020.11.1.pdf available at https://authorea.com/users/376998/articles/493839\%CE\%B5lectrocardiographic-and-electrophysiological-characteristics-of-idiopathicventricular-arrhythmias-with-acute-successful-ablation-at-the-left-ventricle-basalinferoseptum-near-the-mitral-annulus 


\section{Hosted file}

Table 3.2020.11.1.pdf available at https://authorea.com/users/376998/articles/493839\%CE\%B5lectrocardiographic-and-electrophysiological-characteristics-of-idiopathicventricular-arrhythmias-with-acute-successful-ablation-at-the-left-ventricle-basalinferoseptum-near-the-mitral-annulus 